\section{$\underset{\substack{\text { hommes } \\ \text { \& migrations }}}{ }$}

\section{Hommes \& migrations}

Revue française de référence sur les dynamiques

migratoires

$1298 \mid 2012$

France - Algérie, le temps du renouveau

\title{
Un visage en mie de pain : une drôle de madeleine de Proust
}

\author{
Hélène du Mazaubrun
}

\section{(2) OpenEdition \\ Journals}

\section{Édition électronique}

URL : http://journals.openedition.org/hommesmigrations/1588

DOI : 10.4000/hommesmigrations.1588

ISSN : 2262-3353

\section{Éditeur}

Musée national de l'histoire de l'immigration

\section{Édition imprimée}

Date de publication : 1 juillet 2012

Pagination : 134-139

ISSN : 1142-852X

\section{Référence électronique}

Hélène du Mazaubrun, « Un visage en mie de pain : une drôle de madeleine de Proust », Hommes \& migrations [En ligne], 1298 | 2012, mis en ligne le 29 mai 2013, consulté le 22 septembre 2020. URL http://journals.openedition.org/hommesmigrations/1588 ; DOl : https://doi.org/10.4000/ hommesmigrations.1588

Ce document a été généré automatiquement le 22 septembre 2020

Tous droits réservés 


\title{
Un visage en mie de pain : une drôle de madeleine de Proust
}

\author{
Hélène du Mazaubrun
}

1 L'histoire de Frida Rochocz est un trait d'union entre deux continents, entre deux cultures du Nord et du Sud, entre un passé douloureux et un avenir d'espoir. Jeune femme, elle quitte l'Argentine, soumise alors à la dictature militaire, pour s'installer en France. Sa vie durant, elle cherchera à retisser le lien avec son pays natal, un lien que symbolise un petit objet en mie de pain, devenu talisman, qu'elle conserve au fond de sa poche.

2 Argentine, 1959. Frida Rochocz vient au monde dans une famille moderne, recomposée, et devient la benjamine de deux frères. Elle est la fille d'un immigré juif allemand et d'une immigrée espagnole. Sa naissance marque l'enracinement de ses parents sur le nouveau continent, au lendemain de la Seconde Guerre mondiale. Au tournant du XX siècle, nombreux sont les Européens qui fuient en Amérique latine. Les vagues migratoires se succèdent. 1933, Hitler devient chancelier et entame une politique du "redressement national" qui conduit un grand nombre de juifs allemands à quitter leur pays. Le grand-père paternel de Frida est fusillé pour ses opinions et sa religion. Son père, juif de Berlin, est forcé de s'échapper de son pays. Engagé politiquement, il participe en Espagne à la guerre civile, ce qui lui vaut d'être fait prisonnier dans un camp situé au sud de la France. 1936, la dictature instituée par Franco pousse un grand nombre d'Espagnols à embarquer en Amérique latine. La mère de Frida suit ses propres parents dans cet exil en Argentine, en raison de leur militantisme politique. L'histoire de Frida est, de ce fait, empreinte de la dualité de deux cultures, du nord au Sud de l'Europe, héritées des origines parentales. L'Argentine est une terre d'immigration jusqu'en 1945, date de la défaite du gouvernement nazi qui pousse beaucoup d'Allemands à rejoindre l'Argentine ${ }^{1}$. Les parents de Frida Rochocz se rencontrent à Buenos Aires à cette époque, tandis que le contexte politique et économique en Argentine, au lendemain de la Seconde Guerre mondiale, commence déjà à se durcir. L'Argentine dans laquelle grandit Frida est marquée par de fréquents changements de gouvernement, une faible croissance économique et une continuelle pression sociale. 
Deux dictatures militaires s'installent : la "Révolution libératrice" de 1955 à 1958 et la "Révolution argentine" de 1966 à 1973. Anarchistes, les grands-parents de Frida sont tués lors des premières répressions des manifestations. La politique et le militantisme occupent une place centrale dans la famille de Frida. À la maison, ses frères en particulier sont très engagés, tandis que les gouvernements successifs répriment avec violence la résistance péroniste, particulièrement dans les secteurs de la Jeunesse péroniste ${ }^{2}$.

\section{Une vie dans les soubresauts de l'histoire}

Malgré ce contexte politique houleux, Frida grandit dans une famille ouverte: sympathisants, artistes, des gens de toute classe sociale vont et viennent dans le salon. Cependant, commence déjà la "double vie" de Frida, partagée entre l'ambiance festive de la maison et l'éducation rigoureuse de l'école allemande. Malgré les réticences de son père à se lier avec d'autres immigrés allemands, sa mère a insisté pour que sa fille fréquente cet établissement dans l'espoir de lui préparer un meilleur avenir. Frida se réfère à cette période en parlant de "bifurcation mentale entre la Frida à l'école et la Frida à la maison ${ }^{3 "}$. Son enfance prend fin brusquement. Outre la mort de son père qui survient alors qu'elle n'a que 8 ans, le climat politique du pays s'aggrave dans les années soixante-dix jusqu'à toucher directement la famille. Son frère, militant politique, est emprisonné avec sa femme. Frida, âgée de 15 ans, accompagne alors sa mère chaque week-end pour lui rendre visite. "J'étais écartelée entre les week-ends à la maison que nous passions en visite carcérale et la semaine où il fallait faire comme si de rien n'était, aller à l'école allemande et ne rien dire, me comporter comme une fille comme les autres. Pourtant, je sentais dans les rues que le danger grandissait et en prison que les conditions se durcissaient." 1976, un autre coup d'État survient. Le général Videla, renversant Isabel Perón, instaure une dictature militaire qui va durer jusqu'en $1983^{4}$. Ce régime est responsable de la mort ou de la disparition de 30000 personnes et de l'exil de millions d'Argentins. Frida et sa mère sont enlevées et placées dans un camp de détention. "Nous serons relâchées au bout de quelques jours. Sans doute recherchaient-ils quelqu'un d'autre? Mais cela faisait aussi partie d'un mécanisme de la terreur auprès des familles de détenus. À la suite de cet épisode, j'ai compris que je ne pourrais plus retourner voir mon frère en prison." Peu de temps après, son autre frère décède d'une leucémie. Frida, qui n'avait pas encore vécu sa jeunesse, perdait l'espoir de pouvoir un jour la vivre en Argentine: "Je n'avais pratiquement plus de chances de vivre là." Et c'est ainsi qu'à 17 ans, elle prend son destin en main. Après l'obtention de son baccalauréat, elle embarque seule sur un bateau cargo et quitte l'Argentine. Son but est de rejoindre un ami d'école installé en Hollande. Des vingt et un jours de bateau, elle se souvient de pratiquement chaque seconde. Elle se rappelle en particulier la halte à Rio de Janeiro qui lui a fait craindre d'être arrêtée et renvoyée en Argentine en vertu d'une convention signée entre les dictatures argentine et brésilienne. Malgré ce moment d'angoisse, la traversée est douce et portée d'espoir. 


\section{Trouver une place où se (re)construire}

4 1976, Frida débarque en Europe. “J'avais envie de croquer chaque jour à pleines dents et en même temps avec cette douleur à l'intérieur qui n'a pas cessé." En Hollande, elle ne trouve pas sa place et décide d'aller à Berlin, dans la ville de son père. Là, elle tente de retrouver quelques traces dans les archives. Elle arpente les rues comme pour remonter le fil de l'histoire. Cependant, il ne lui reste plus de famille en Allemagne. Déracinée, Frida ne sait pas où s'installer : “Je ne savais pas où rester (...). Je n'ai voyagé que parce que j'étais obligée. Je ne suis pas venue de mon propre gré. Je n'avais aucune envie d'aller nulle part. Je voulais rester dans mon pays, mais je ne pouvais pas. Jusqu'à maintenant, je dois clore cette étape qui n'est pas encore fermée." Frida va donc multiplier les voyages, pour finalement s'installer à Barcelone. Sa mère l'y rejoint après que son fils, emprisonné, a été déclaré "disparu". Cette période est difficile pour Frida. Elle comprend qu'elle ne retrouvera jamais la relation qu'elle avait avec sa mère avant son départ pour l'Europe: “Ma mère ne s'est jamais vraiment remise psychologiquement. Elle pensait qu'elle venait me sauver, mais en fait, c'est moi qui ai dû la soutenir. Ma mère était une femme très courageuse, une Espagnole de naissance, elle a travaillé. (...) Mais plus rien ne pouvait la toucher et elle m'a toujours rejetée, à cause de cela. Je crois que cela fait partie d'un syndrome de ce qu'ont pu vivre pas mal de survivants, comme ceux de la Shoah. Finalement, la personne qui leur reste, ils ne peuvent plus l'aimer, par peur de la perdre." Frida décide donc de retourner vivre à Berlin. Sur le chemin de ses allers-retours entre Berlin et Barcelone se trouve Paris. Un peu par hasard, Frida va donner des cours d'allemand dans une école privée de la tour Montparnasse. Aujourd'hui, elle s'amuse de la situation: "Une Argentine qui enseigne l'allemand à Paris, qui plus est avec un accent des années vingt, celui de mon père !" Frida a 20 ans, lorsqu'elle pose enfin ses valises quelque part, en France, entre deux pays qui lui rappellent ses origines et sa vie en Argentine. Si elle construit rapidement sa vie personnelle ("tout de suite je me marie, tout de suite j'ai un enfant"), elle a plus de mal avec sa vie professionnelle. Elle explique cela par le fait qu'elle n'a pas eu d'adolescence. C'est pourtant important, rappelle-t-elle, "ce moment de latence propice pour se réaliser ensuite dans le monde professionnel. Du coup, je multiplie les boulots alimentaires auxquels je m'identifie très peu, mais de bon cœur". Bien qu'elle reprenne des études supérieures en lettres et en sciences humaines, elle se voit contrainte de faire passer en priorité sa vie personnelle et l'éducation de ses deux filles. "Je ne demandais rien à personne !" De son indépendance, Frida semble fière. Exilée politique, elle ne bénéficie pas pour autant du statut de réfugié ; elle n'en a pas fait la demande. En Allemagne, elle avait obtenu la nationalité allemande grâce à la nationalité de son père, ce qui lui a permis de vivre, dit-elle, "comme citoyenne européenne". Mais en France, sans statut de réfugié, elle ne profite d'aucun accompagnement social. Aujourd'hui, hasard de la vie, elle travaille dans un service social. Elle aide des personnes en difficulté à construire leur projet. Frida sait qu'elle aurait pu recevoir une aide équivalente lors de son arrivée en France. Pourtant, elle n'a jamais entamé les démarches: "Je ne me suis jamais considérée comme pauvre ou immigrée. Je savais qu'il existait des assistantes sociales, mais ce n'était pas pour moi." Et de répéter : "Je ne me suis jamais sentie immigrée. Pour moi, les États ce sont des constructions artificielles, économiques et politiques (...). Le monde est pour moi très vaste, très ouvert. Dans ma tête, il n'y a pas de frontières." 


\section{Le temps du témoignage}

5 Avant que Frida ne retourne en Argentine, il s'écoule plus de dix ans. "Le retour en Argentine a été très long, car j'ai attendu la fin de la dictature. (...) J'avais perdu le contact avec tout le monde." Le déclic est provoqué par un copain de lycée qui la retrouve à Paris et l'encourage à y retourner. "C'est très douloureux de revenir dans un endroit où l'on n'est pas chez soi (...) tellement bouleversant de marcher dans les rues, de sentir les odeurs..." C'est à partir des années deux mille qu'elle retourne plus régulièrement en Argentine: "Chaque voyage, c'était pour rechercher des gens, les survivants, pour essayer de retrouver les choses." En 2005, les deux lois d'amnistie qui ont empêché la justice argentine de suivre son cours sont déclarées inconstitutionnelles 5 . Les procès débutent. Frida est appelée à témoigner à trois d'entre eux. Le premier concerne la disparition de son frère. "C'était très beau du point de vue des émotions, parce que j'ai retrouvé des gens que je n'avais pas vus depuis très longtemps, certains depuis la cour de la prison, qui m'ont serrée dans leurs bras en me disant : Tu étais si petite." Le retour en Argentine est donc mêlé de "souvenirs qui sont les souvenirs d'une jeune fille adolescente", et du sentiment d'un "devoir de reconstruire ces choses, pour pouvoir les porter en justice". Dans cette recherche de souvenirs, Frida utilise entre autres Facebook. C'est justement un camarade de lycée qui, ayant essayé en vain de la retrouver en Europe lors de l'un de ses voyages, l'avait invitée sur ce réseau social. Elle comprend qu'elle peut ainsi renouer avec les anciens de l'école allemande. Alors qu'elle était réticente dans un premier temps, cet ami l'y encourage. Frida se rappelle cet échange: “Tu serais étonnée si tu rencontrais les gens... Tout le monde se souvient de toi... Frida, il faut que tu saches une chose : nous, nous avions tous des familles très coincées, et toi, tu avais un monde très différent. Quand on allait chez toi, c'était un monde extraordinaire.' Et moi, je pensais que ma maison était tellement petite à côté de la leur. 'Frida, quand tu entrais, la liberté entrait avec toi."'Depuis, Frida est entrée en contact avec ses camarades d'enfance. Elle attend son prochain voyage en Argentine (pour deux autres procès) : “J'aimerais oser les voir un peu plus et franchir le prochain pas pour essayer de guérir cette plaie qui n'en finit pas de cicatriser." La blessure est bien là, encore, gravée mentalement et physiquement : “J'ai fait de l'asthme très fort, car le corps n'a plus supporté que je ne parle pas. J'avais la sensation de vivre une fausse vie, une double vie, comme s'il y avait une Frida qui vivait là-bas et une autre qui vivait ici. Il faut dire que la prise de parole est toujours délicate sur les histoires traumatiques."

\section{La madeleine de Frida}

6 Si Frida s'est longtemps tue, elle gardait auprès d'elle un objet qui la rattachait à son passé et à ceux qu'elle avait laissés derrière elle. Il s'agit d'une statue de mie de pain qu'elle a décidé de donner au Musée national de l'histoire de l'immigration, à Paris. Cette curieuse "madeleine de Proust" est en fait un cadeau de son petit ami de l'époque, un ami de son frère qui avait été emprisonné comme lui. Ce type de présent est souvent réalisé par les détenus. Cet objet a suivi Frida toute sa vie, au fondde sa poche. En réalité, elle n'a réussi à s'en séparer qu'à la naissance de ses enfants: "Les enfants m'ont beaucoup aidée. Je ne dirais pas pour m'enraciner en France, mais pour m'enraciner dans la vie." Sans doute peut-elle se détacher de cet objet, parce qu'elle a 
retrouvé cet ami, de longues années après son exil. Lorsqu'elle le rencontre, elle lui demande : "Te souviens-tu de cet objet?" La réponse est négative. Ce visage en mie de pain devient alors, dans l'esprit de Frida, le symbole des trajectoires de mémoire et de leur subjectivité. En effet, cet ami est resté tant d'années en prison qu'il ne se rappelle plus ce moment ni ce cadeau. Pour Frida, cet objet cristallise le moment de son départ. "Cela me semble important dans la vie de comprendre que l'on peut revenir sur son histoire, sur le passé, et qu'on ne l'a pas tous vécu de la même façon." Autrement dit, cet objet est aussi une fenêtre qui s'ouvre sur la mémoire et qui permet de rebattre les cartes de l'histoire, de réviser les points de vue et son propre récit de vie. Justement, si Frida a gardé ce cadeau de mie de pain, c'est qu'il révèle une partie de sa personnalité : un visage humain, une matière fragile, une relation aux autres et au passé. Elle le décrit ainsi : “C'est une tête. Je pense que ce prisonnier à l'époque ne connaissait pas Le Cri de Munch, mais il a quelque chose de ce Cri, car il a les yeux qui sont faits à peine, et il a une bouche grand ouverte qui appelle. Il crie, il a une voix. C'est une toute petite tête qui tient dans la paume de la main. Dans ma poche, je mettais toujours le pouce et l'index sur l'espace des yeux, et la bouche restait ouverte." Enfin, sur ce visage de mie de pain s'ajoute une histoire qui avait été racontée à Frida dans sa jeunesse : Un jeune homme fuit le ghetto de Varsovie pour entrer en contact avec des partisans. Au moment de son départ, une personne lui remet un petit paquet emballé dans du tissu en lui disant: "Il y a un morceau de pain à l'intérieur, mais promets-moi que tu ne le mangeras que lorsque vraiment tu n'auras plus aucune force." Dans sa fuite, l'homme rencontre bien des difficultés. Il doit se cacher, lutter contre les intempéries, vaincre la fatigue et la faim. Il est souvent tenté de manger le morceau de pain, mais il résiste. Arrivé au bout de sa course, il est enfin accueilli et s'écroule d'épuisement. Il peut se reposer enfin et décide de se rassasier du morceau de pain. Mais lorsqu'il déplie le carré de tissu, il découvre un morceau de bois à l'intérieur. Frida conclut cette histoire ainsi : “Je crois que ce morceau de bois l'a porté, lorsqu'il avait besoin d'espoir. Je pense que la statuette de mie de pain, c'est la même chose dans mon histoire. Je me sens portée par des choses que je dois encore finir de faire. Peut-être que ma vie va commencer après."

\section{NOTES}

1. La présence d'Allemands en Argentine remonte au XIX ${ }^{\mathrm{e}}$ siècle. Dès 1870 , lors de la guerre franco-allemande, beaucoup d'Allemands étaient venus s'installer dans les terres vides d'Argentine. Beaucoup encore vinrent à la suite de la défaite de 1918. Les conditions de vie sous la République de Weimar étant difficiles, un grand nombre se décida à rejoindre un oncle ou un cousin qui avait immigré auparavant en Argentine. En 1945, l'exode est massif, qu'il s'agisse des familles compromises dans le régime nazi ou des familles qui espéraient une vie meilleure qu'en RDA.

2. Juan Domingo Perón, militaire et homme politique, devient le président de la nation argentine de 1946 à 1955 et de 1973 jusqu'à sa mort en 1974, date à laquelle lui succède sa troisième épouse, Isabel Perón. 
3. Entretien réalisé le 30 mai 2012 au Palais de la Porte Dorée, Cité nationale de l'histoire de l'immigration, Paris ; de même que toutes les citations de Frida dans ce texte.

4. Jorge Rafael Videla, général et homme politique, dirige l'Argentine et la " guerre sale ", après le coup d'État militaire du 24 mars 1976 qui destitua Isabel Perón. En 1981, il céda la présidence de la junte au général Roberto Eduardo Viola. Il est placé en résidence surveillée au retour de la démocratie en 1983, puis condamné à la prison à perpétuité lors du procès de la junte en 1985. En 1989, il est amnistié par le président Carlos Menem, avant que son dossier ne soit rouvert en 2007. En 2010, il est condamné à la prison à vie.

5. La loi 23456 du Point final (Punto final) est une loi argentine promulguée le 24 décembre 1986, sous la présidence de Raúl Alfonsín, qui interdisait les poursuites au pénal contre les crimes commis lors de la dictature argentine pour toute plainte dépassant le délai de prescription de 60 jours fixé à compter de la promulgation de la loi. La loi amnistiait de facto toute détention arbitraire, torture ou assassinat commis jusqu'en 1983, année de la fin de la dictature militaire. Seul le cas des "bébés volés" sortait de l'application de la loi, disposition spécifiée par l'article 5. Celle-ci fut suivie, l'année suivante, par la loi d'obéissance due, qui protégeait les militaires de rang inférieur au nom du principe hiérarchique de l'armée, puis par les amnisties décrétées par Carlos Menem en 1989-1990. L'ensemble de cette réglementation est connu sous le nom de "lois d'impunité".

\section{AUTEUR}

\section{HÉLÈNE DU MAZAUBRUN}

Chargée des collections, responsable de la Galerie des dons, Musée de l'immigration, CNHI. 